\title{
Effect of Symlet Filter Order on Denoising of Still Images
}

\author{
S. Kumari ${ }^{1}$, R. Vijay ${ }^{2}$ \\ ${ }^{1}$ Department of Physics, Banasthali University - 304022, India \\ sarita.kumari132@gmail.com \\ ${ }^{2}$ Department of Electronics, Banasthali University-304022, India
}

\begin{abstract}
In wavelet-based image coding the choice of wavelet is crucial and determines the coding performance. The current compression techniques involve time taking procedures to find out the optimal basis. In this paper, we show that Sym 5 represents the best results for compression and denoising of natural images. The performance of compression is measured in terms of CR and PSNR and it is analyzed that with increase of filter order PSNR value increases but visual quality of the compressed image degrades rapidly.
\end{abstract}

\section{KEYWORDS}

Optimal Basis, Filter Order, Compression Ratio, Peak Signal to Noise Ratio, Symlet Wavelet

\section{INTRODUCTION}

Digital image compression is used to reduce the number of bits required to store an image in computer memory and/or transmit it over a communication link. The main focus of the research in this area is to enhance the performance of the current digital image compression method. Image compression minimizes the size in bytes of a graphics file without degrading the quality of the image to an unacceptable level. The reduction in file size allows more images to be stored in a certain amount of disk or memory space. It also reduces the time necessary for images to be sent over the internet or downloaded from web pages.

While compressing an image de noising is one of the challenging tasks for researchers because noise removal introduces artifacts and causes blurring of the images. Wavelet thresholding is the promising method for image denoising while compression. It reduces the blocking artifacts as it considers the image as a whole while considering block by block as the case of DCT. The aliasing distortion can be reduced with a proper choice of wavelet filters.

Wavelet families are mainly distinguished into two categories: Orthogonal and Biorthogonal. Orthogonal wavelet families are, Daubechies, Coiflet and Symlet. The performance of wavelet based coding depends on the wavelet decomposition structure. Before employing a computationally intensive procedure for finding the optimal basis, it will be helpful to know, a priori, if appreciable gain can be achieved by using the optimal basis over known good wavelets [1]. In this paper, we try to find out that what should the optimal filter order be for most images? However, it has been shown that symlet wavelet family gives better performance 
with increased compression ratio (CR) and energy retained (ER) [2] [3]. The work is applied to find out the optimum filter order for Symlet wavelet family.

\section{CHOICE OF WAVELET TYPE AND ORDER}

There are a number of basis functions that can be used as the mother wavelet for Wavelet Transformation. Since the mother wavelet produces all wavelet functions used in the transformation through translation and scaling, it determines the characteristics of the resulting Wavelet Transform. Therefore, the details of the particular application should be taken into account and the appropriate mother wavelet should be chosen in order to use the Wavelet Transform effectively. The wavelets are chosen based on their shape and their ability to analyze the signal in a particular application.

An important property of wavelet analysis is perfect reconstruction, which is the process of reassembling a decomposed signal or image into its original form without loss of information [4]. To achieve a high compression rate, it is often necessary to choose the best wavelet filter bank and decomposition level, which will play a crucial role in compressing the images. The selection of wavelet filters plays a crucial part in achieving an effective coding performance, because there is no filter that performs the best for all images. The choice of optimal wavelets has several criteria. The main criteria are:

\section{Orthonormality}

2. Filter Length

3. Vanishing order or moment

4. Smoothness

5. Decomposition level

6. Regularity

To determine the effect of the choice of the wavelet filter order, we compare the performance of some known wavelet families. It is found that symlet filter gives good performance for natural images. Then we compare the coding performance of wavelets of various orders within the same family. In this study, we have used the symlet-2,3,4,5,6,7,8,9,10,11,12 taps.

\section{COMPRESSION QUALITY PREDICTION}

Numerous papers introduce various objective and subjective metrics; our work deals with the following criteria: 


\subsection{Compression Ratio (CR)}

Compression ratio is an important parameter to know the amount of compression [5]. It is defined as the ratio of original image size to the compressed image size

$$
\text { Comprassion_ratio }=\frac{A}{B} \times 100
$$

Where $\quad A=$ Number of Bytes in the original data set

$$
\mathrm{B}=\text { Number of Bytes in the Compressed data set }
$$

\subsection{Peak Signal to Noise Ratio (PSNR)}

The peak signal to noise ratio (PSNR) given below where $2^{\mathrm{R}}-1$ is the maximum gray level number [6].

$$
P S N R=10 \log _{10} \frac{\left(2^{R}-1\right)^{2}}{M S E} d b=20 \log _{10} \frac{2^{R}-1}{R M S E} d b
$$

When the original and the decompressed images are identical, the PSNR tends towards infinity. In most cases, a PSNR greater than $30 \mathrm{db}$ is considered as leading to a correctly reconstructed image.

\section{PROPOSED ALGORITHM}

I. Load the image from the user.

II. Apply 2D DWT using symlet wavelet over the image.

IV. Compress the image by factors of $1 / 10,1 / 20,1 / 30,1 / 40,1 / 50,1 / 60,1 / 70,1 / 80$ $1 / 90$ and $1 / 100$ respectively.

VI. MSE and PSNR are calculated for different threshold values.

VII. Repeat the same process for various images with various filter orders and compare its performance.

\section{RESULTS AND DISCUSSION}

In our experiments, we used the gray scale images, of size 256X256 and measured the compression ratio and the PSNR of the compressed image. The quality of compressed image depends on the number of decompositions. 
Advanced Computing: An International Journal ( ACIJ ), Vol.3, No.1, January 2012

Table-1: CR and PSNR Values for Symlet Family of gray scale Images

\begin{tabular}{|l|c|c|c|c|c|c|c|c|c|c|}
\hline \multirow{3}{*}{ Image } & \multicolumn{2}{|c|}{ Sym4 } & \multicolumn{2}{c|}{ Sym6 } & \multicolumn{2}{c|}{ Sym8 } & \multicolumn{2}{c|}{ Sym 10} & \multicolumn{2}{|c|}{ Sym 12} \\
\cline { 2 - 10 } & CR & PSNR & CR & PSNR & CR & PSNR & CR & PSNR & CR & PSNR \\
\hline lena & 99.958 & 42.360 & 99.964 & 42.306 & 99.969 & 42.312 & 99.971 & 42.253 & 99.974 & 42.242 \\
\hline bird & 99.981 & 49.306 & 99.986 & 49.085 & 99.989 & 48.884 & 99.990 & 48.936 & 99.992 & 48.887 \\
\hline goldhill & 99.937 & 42.069 & 99.951 & 42.016 & 99.960 & 42.036 & 99.965 & 42.015 & 99.971 & 42.047 \\
\hline mandrill & 99.939 & 41.796 & 99.947 & 41.779 & 99.954 & 41.823 & 99.958 & 41.760 & 99.963 & 41.760 \\
\hline peppers & 99.934 & 42.058 & 99.947 & 42.050 & 99.956 & 42.003 & 99.960 & 41.964 & 99.966 & 41.958 \\
\hline frog & 99.926 & 41.15 & 99.93 & 41.090 & 99.945 & 41.126 & 99.951 & 41.076 & 99.957 & 41.041 \\
\hline camera & 99.974 & 48.520 & 99.980 & 48.377 & 99.984 & 48.287 & 99.986 & 48.397 & 99.988 & 48.225 \\
\hline
\end{tabular}


Advanced Computing: An International Journal ( ACIJ ), Vol.3, No.1, January 2012
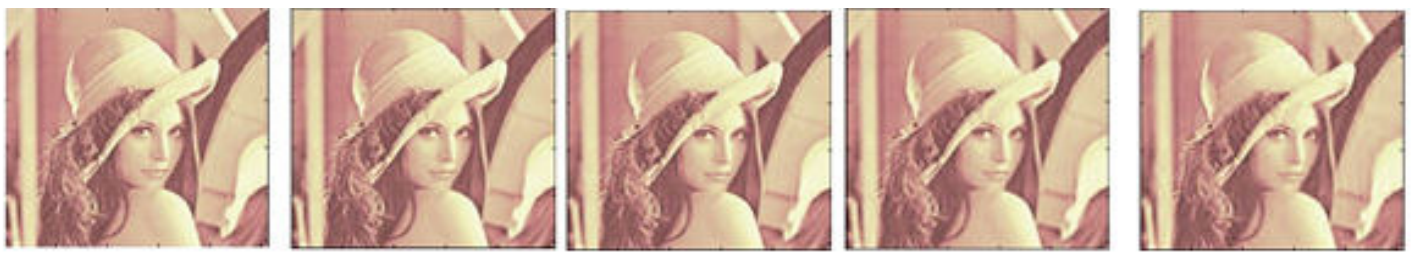

Fig l: original and reconstructed lena images for level $1,2,3$ and 4

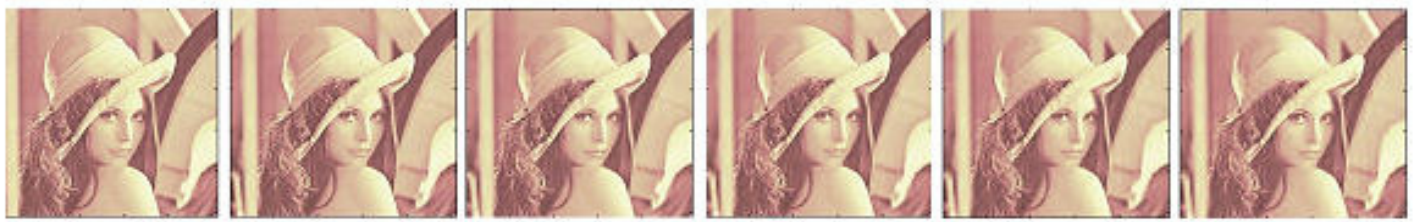

F ig 2: original and reconstructed lena images at threshold $20,40,60,80$ and 100

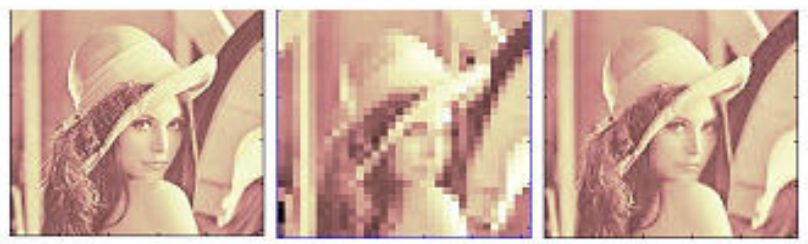

Fig 3: original, decomposed and reconstructed lena images with Symlet 4 tran sfo $\mathrm{rm}$

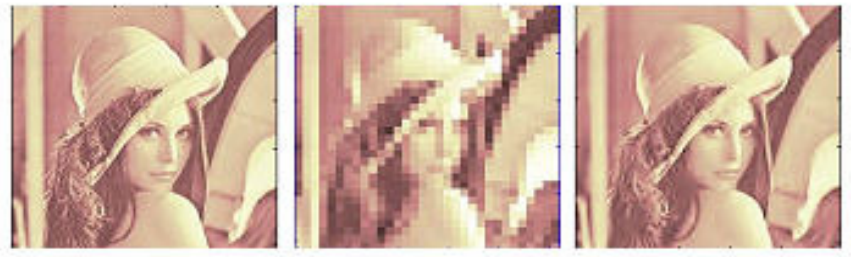

F ig 4: original, decomposed and reconstructed lena images with Sym let 8 transform
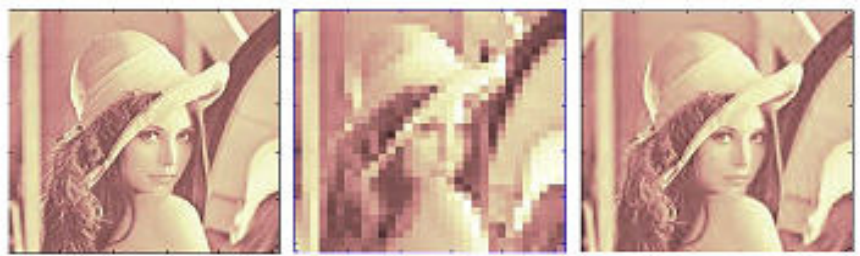

Fig 5: o riginal decomposed and reconstructed lena images with Symlet 12 tran sform

Figure1 shows the original and reconstructed lena image for level 1,2,3 and 4. It can be seen easily that the quality of compressed image depends on number of decompositions because HVS is less sensitive to removal of smaller details. Figure2 shows the effect of threshold on the quality of reconstructed lena image. It is seen that quality of reconstructed image degrades rapidly with increasing threshold. Figure $3,4,5$ shows the original, decomposed and reconstructed lena image with symlet 4 , symlet 8 and symlet 12 respectively. The visual quality of reconstructed image is better in case of symlet 4 as compare to higher order filters. For higher 
threshold values quality of images compressed using sym4 slowly degrades while quality of sym12 compressed images deteriorate rapidly.

Comparison of PSNR of lena, bird, mandrill, pepper, camera and goldhill images with Symlet DWT using 4, 8, 12-tap is shown in fig 6. For higher compression ratios quality of images compressed using sym 4 slowly degrades whereas for higher orders it degrades rapidly. Thus it can be concluded that the compression performance of sym 4 is superior to that of sym 12 and the visual quality of reconstructed image is better even if at the same threshold.

To examine the influence of the length of a wavelet filter to image quality, we changed the number of taps in symlet wavelet. PSNR and CR values for 2,4,6,8,10 and 12 taps are contained in Table 1. Larger number of taps does not imply better PSNR and visual picture quality. Hence, it is always necessary to select the optimal threshold value to get higher compression and to achieve minimum loss of images [2]. Number of decompositions in these examples is chosen to be optimal for corresponding wavelet, which is found 4 in case of each image with threshold at 10 .

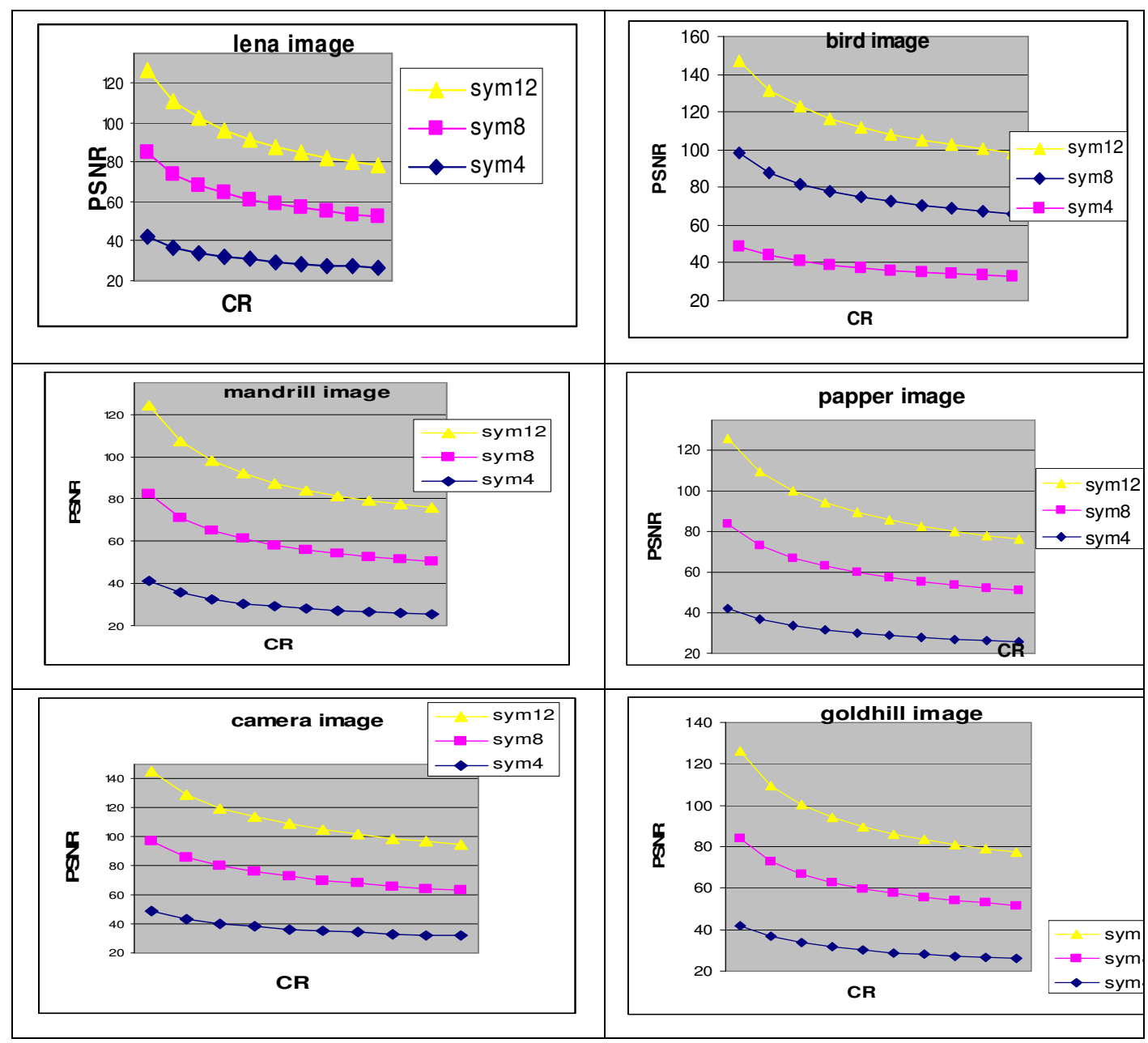

Figure 6: Results of PSNR of mage lena, bird, mandrill, pepper, camera, goldhill with symlet wavelet filter of order 4,6,8,10 and 12 


\section{CONCLUSION}

Here comparison of Symlet filter order is given with various threshold values at different level of decompositions. Results show that as the filter order is going to increase, compression is increasing, but visual quality of compressed image is degraded. Thus it is analyzed that the order of a wavelet filter is important in achieving good coding performance. Moreover, best visual quality is found for Sym 5 wavelet with good compression ratio.

\section{REFERENCES}

[1] S. Kumari and R. Vijay, (2011), “Analysis of Orthogonal and Biorthogonal Wavelet Filters for Image Compression", International Journal of Computer Applications, Vol. , No., pp17-19.

[2] S. Kumari, R. Vijay, (2010), "Image Quality Prediction by Minimum Entropy Calculation for Various Filter Banks", International Journal of Computer Applications, Vol. 7, No. 5, pp 27-34.

[3] S. Kumari, R. Vijay \& V. S. Meel, (2009), "Selection Of Wavelet Basis For Compression of Natural and Line-Based Images”, International Journal of Information Retrieval, Vol. 2, No. 1, pp 1-5

[4]Brittain,N.J.\&El-Sakka,M.R., (2007), "Grayscale true two-dimensional dictionary-based image compression", J. Visual Communication and Image Representation, Vol 18, pp 35-44

[5] G.K. Kharate, V.H. Patil, N.L. Bhale, "Selection of Mother Wavelet For Image Compression on Basis of Nature of Image”, Journal of Multimedia, Vol2,No6, November 2007.

[6] Abla Kourzi, Danielle Nuzillard, Gilles Millon, Frédéric Nicolier, "Quality Estimation In Wavelet Image Coding”, CReSTIC, Université de Reims Champagne Ardenne.

\section{Authors}

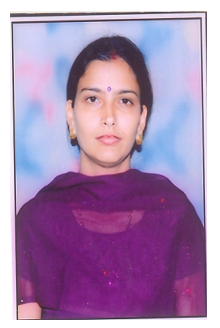

Mrs. Sarita Kumari received her Ph.D. (Image Processing) degree from Bansthali University in 2011. Since 2005 she is working as a faculty in the Department of Physics at Banasthali University. Her main research area includes Image processing, wavelet theory and material science. She has guiding six MS theses in these areas. She has published seven papers in International and National Journals and also published around twenty papers in International and National Conferences conducted bothin India and abroad. She is life member of International Association of Engineers (IAENG) and World Academy of Science, Engineering and Technology.

Dr Ritu Vijay received her master's degree in Electronics from Banasthali University in 1997. She obtained her Ph.D. (Signal Processing) degree from Bansthali University in 2005. Currently she is head and Associate Professor in Department of Electronics, Banasthali University. Her main research area includes Signal Processing, Image processing and Nureal Networks. She is twelve Ph.D. theses in these areas. She has published several papers in International and National Journals and also published around thirty papers in International and National Conferences conducted bothin India and abroad. She is life member of Indian Unit for Pattern Recognition and Artificial Intelligence (IUPRAI). 